\title{
Session 21.4 - World Heritage and the Protection of Working Observatory Sites
}

\author{
Clive Ruggles \\ School of Archaeology and Ancient History \\ University of Leicester, Leicester LE8 0PJ, United Kingdom \\ email: rug@le.ac.uk
}

\begin{abstract}
This joint session between FM21 and FM2 ("Astronomical Heritage: Progressing the UNESCO-IAU Initiative") focused upon the need to preserve the dark skies necessary for the continued functioning of the world's leading optical observatories and whether, if some of the sites concerned could be inscribed on UNESCO's World Heritage List, this could help achieve this objective. Among the main issues addressed were: is a WHL inscription feasible in the first place? how could the strongest case for inscription be made? what progress has been made towards doing this? and what other effects might a WHL inscription have and would they all be desirable to astronomers? Addressing such issues involves not only scientific but also heritage and political considerations.
\end{abstract}

Keywords. Astronomical heritage, World Heritage, Leading observatories, High-mountain observatories

\section{Introduction}

Dark skies cannot of themselves be recognised under the World Heritage Convention. However, light pollution not only affects night sky quality but also affects the integrity of other resources and, indeed, whole ecosystems. It is also linked to the issue of energy waste through lighting. These factors affect the sustainable management of both cultural and natural sites, including existing and potential World Heritage Sites.

This - the first of two joint sessions with FM2 ("Astronomical Heritage: Progressing the UNESCO-IAU Initiative") dealing with preserving dark skies and protecting against light pollution in a World Heritage framework - focused upon working observatory sites.

The first four presentations were all by contributors from Chile, which is playing a vital role in the "Windows to the Universe" project, one of the main nomination projects being advanced within the UNESCO-IAU Astronomy and World Heritage Initiative. The last of the Chilean contributions, together with a further three papers, described efforts to protect observatories from light pollution and/or radio frequency interference at various leading observatories. The remaining paper outlined, both from an astronomical and a heritage perspective, a practical approach to the recognition of Dark Sky places as possible World Heritage sites using the Pic du Midi Observatory as a case study.

\section{The protection of dark skies in Chile}

Malcolm Smith (The AURA Observatory in Chile-part of the IAU/UNESCO Extended Case Study) described the "Windows to the Universe" concept and how, as is hoped, it could serve as the basis for an international serial (multi-site) nomination for the inscription on UNESCO's World Heritage List of outstanding, high-mountain, groundbased, observatory sites developed over the period 1870-2000. An Extended Case Study 


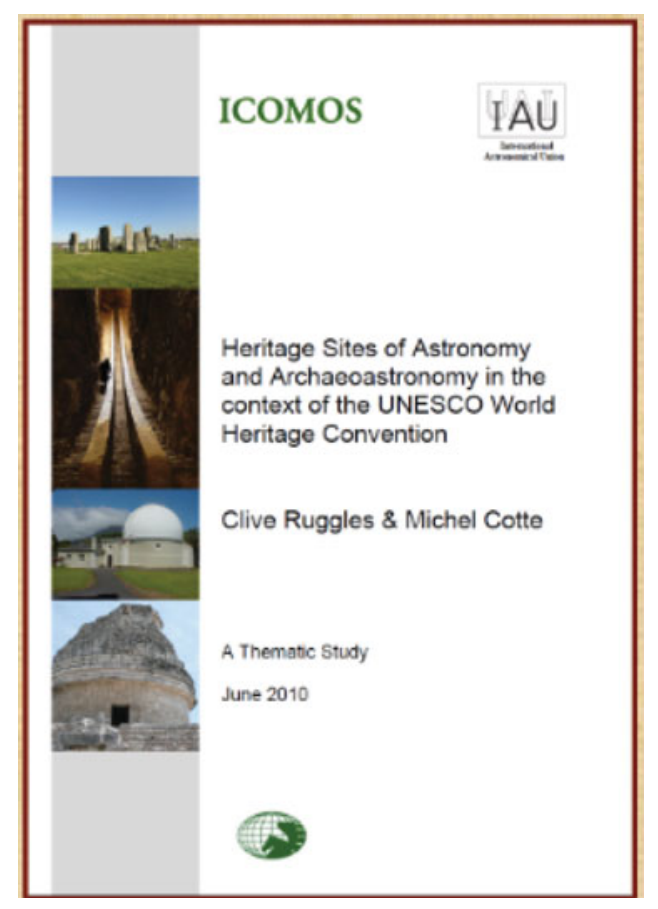

Figure 1: The publication of the UNESCO-IAU Portal to the Heritage of Astronomy

for AURA-O as a "Window to the Universe" has been prepared for inclusion in the second ICOMOS-IAU Thematic Study on astronomical heritage, to be published later this year (Ruggles \& Cotte 2015), and has been published on the UNESCO-IAU Portal to the Heritage of Astronomy (http://www.astronomicalheritage.net/index.php/showentity?identity=000059\&idsubentity=005).

AURA-O includes the Cerro Tololo InterAmerican Observatory (CTIO) established in 1962 as the first of the major international observatories to be installed in Chile. The future of AURA-O now includes the Large Synoptic Survey Telescope (LSST). This Extended Case Study has provided the context for the development of possible initiatives to protect a variety of sites in Chile (e.g. Tololo, Pachón, La Silla, Las Campanas, Paranal, Armazones and Chajnantor) for their historical and scientific value to humanity. The dark skies and ideal weather patterns of northern Chile, along with its location in the southern hemisphere, have made this area of the world a major centre for astronomical facilities.

Following this presentation, Ambassador Gabriel Rodríguez G-H, Director for Energy, Science \& Technology and Innovation in the Chilean Ministry of Foreign Affairs, was invited to give an example of a government's response to this initiative and its implementation in practice. His report is quoted here in detail:

\section{Chile: an experience of government policy for the protection of "dark skies"}

\subsection{The context}

Astronomy has been increasingly present in Chile during the last fifty years. As of today, almost $50 \%$ of all the optical and radio-astronomical observational capabilities on the entire planet are located in the arid northern regions of our country. Considering the new big astronomical projects recently inaugurated (ALMA), or planned to be built in 
Chile in the next five to ten years (LSST, GMT, TAO, CCAT), by 2022-25 our country will host almost three quarters of the world's astronomical observation capabilities.

Just in the last decade, Chile has started to be fully aware of this exceptional situation and its consequences, in terms both of opportunities and responsibilities.

\subsection{Why Chile?}

For astronomers, it is pretty obvious that a clean and dry atmosphere, combined with high-altitude sites, sparsely populated areas, and dark skies, all of which exist practically year round, is an ideal mix of conditions for observing the universe. Adding the location in the southern hemisphere, where observations of our galaxy's center and the rest of the universe are better and easier, Chile's northern regions are a privileged place for optical and radio-astronomical observations.

In this sense, Chile has a "natural resource" - clean skies - no different from our resources of copper if we view them from an economical perspective. Clean and dark skies are a natural resource that we can rent, gaining a financial return, or "exploit", extracting some added value from it.

Since the 1960s, when international astronomical activities began in Chile, our country has facilitated the installation of scientific projects in the "Norte Chico" (350 km north of Santiago) and the Atacama desert. In that sense, and initially not being fully aware, the government has developed a "policy" for attracting astronomy projects to our country.

\subsection{Chile as a "natural lab"}

The Atacama desert represents for astronomy what Antarctica does for polar and subantarctic science, the Humbolt current for oceanography, the Cordillera de los Andes for vulcanology, and the geothermal resources and the geological Andean faults for seismology. Chile has unique natural conditions. We call them "natural labs" (a name coined by Chilean scientist José Miguel Aguilera), among which astronomy is the best known. This makes our country an exceptional place for scientific research.

All this represents a big opportunity — "an astronomy platform" for our country —and simultaneously a very serious responsibility: how to protect these "natural labs" for humankind's solace, curiosity and progress.

\subsection{Government astronomy policy}

Chile has a responsibility to preserve these exceptional sites for humanity, and we are determined to be world leaders in the challenge of protecting "dark skies" for the abovementioned purposes.

With that in mind, the Chilean government has developed a four-tier policy which combines our national interests with global ones. We seek to answer the question: "how can we create virtuous connections between astronomy and national development?"

An obvious answer is Astronomy as a Science. We already have a community of more than a hundred Chilean astronomers, and around the same number of future scientists in the pipeline. We are supporting the development of science, and astronomy is a key scence. Today, around $10 \%$ of the papers published as a result of research carried out at the telescopes installed in Chile have Chilean scientists as authors.

The second pillar is Astroengineering. We can view all the astronomical observatories in Chile as scientific labs, but also as a gigantic engineering school for big-data technologies, software development, telecommunications, robotics, optics, electromechanical devices, anti-seismic engineering, electronics, new materials, and so on. Access to highend technologies that can facilitate the identification of opportunities for development and for training have advanced human capital in key areas for our country, enabling it 
to jump ahead to reach development. Robotics today is central to unmanned mining, big data for genetics or geology, and optics for solar energy, just to mention a few examples.

Education and Culture is the third pillar. Astronomy is an attractive niche to entice young students into science, and science is central for development. There is no technological innovation and social progress without science. At the same time, astronomy is more than just science. It is culture. Astronomy has to do with understanding who we are and where we are. These are deep questions whose answers can illuminate personal daily lives and societies.

Finally, the Involvement of Society is crucial. Government can and must play a central role in identifying opportunities around astronomy, but in the end society itself is the key actor in taking this challenge in hand. Government has the duty to involve citizens in protecting the natural resource of dark and clean skies that is the basis for the human right to observe the stars with the naked eye. And it is part of the citizens' duty to protect skies not only as a natural lab for astronomy, but also as places for "astrotourism" and solace.

\subsection{Protecting "dark skies"}

The Chilean government is working in several areas to implement this astronomy policy.

- The Ministry of Foreign Affairs is a "one-stop" contact entry point for international astronomical consortia looking for a place in Chile.

- International Observatories can be granted "diplomatic status".

- Clearly regulated land property access and long-term concessions for scientific installations.

- Declaration of "scientific protected areas" and buffer zones to isolate the observatories from industrial (and particularly mining geothermal) activities, including wavelength interference.

- Infrastructure facilitation.

- Clear rules and technical norms for public lighting, and enforcement of legal capabilities.

- Environmental regulations for dust pollution.

- Energy supply. Energy generation and transmission in Chile is privately owned, but government can help create the best conditions for facilitating the energy supply to scientific observatories.

Because we are convinced that the natural labs we have are not permanent realities, protecting clean and dark skies, in the case of astronomy, is one the main objectives of this policy.

The UNESCO World Heritage Programme is looking to develop a new area of World Heritage: places that have universal value based on their outstanding scientific activities and discoveries.

Based on the definitions and objectives of our own astronomy policy and the opportunity that UNESCO opens up to us, Chile has taken the decision to pursue the declaration of World Heritage through UNESCO for its astronomical sites. There is a long road ahead but at the same time the whole process is an opportunity to involve society in this quest. Involvement is a two way process. On one side is a government responsibility to develop awareness between citizens of the value we already have in our country. On the other side is the responsibility of the Observatories to become involved in local realities and communities and to show the social value of what they are doing. Education and outreach are central to this.

There are many intermediate steps and possible paths. We can move ahead with a local declaration (involving only Chile) or a serial one, at an international level. We can 
select groups of astronomical sites based on their similar scientific characteristics or based on historical features. In all cases, however, it is the need to demonstrate Outstanding Universal Value that is the key element in determining the possibility of a World Heritage Declaration.

\subsection{Conclusions}

Chile's astronomy policy, the four pillars, is an example of how the government can support international astronomical research through protecting our privileged natural labs, while at the same time identifying how astronomy can be a key factor in pursuing development objectives that can have clear and effective social impact on our citizens.

Ambassador Rodríguez's report drew an enthusiastic response from several contributors in the ensuing discussion, who noted with approval the positive attitude and constructive approach taken by the Chilean government towards international astronomical research and maintaining the dark skies upon which it depends.

The following report by Chris Smith et al. (Site protection efforts at the AURA Observatory in Chile) described efforts over the past 20-30 years to highlight the importance of site protection at the AURA Observatory site in northern Chile through education and public outreach as well as through more recent promotion of IDA certifications in the region and support for the World Heritage initiatives. The site, which includes both Cerro Tololo and Cerro Pachón, has been operational for over 50 years, and hosts more than 20 operational telescopes, ranging from small projects with $0.4 \mathrm{~m}$ telescopes to the Blanco 4m, the SOAR 4.1m, and the $8 \mathrm{~m}$ Gemini-South telescopes, with construction of the Large Synoptic Survey Telescope (LSST) having recently begun.

The AURA-O faces a variety of challenges to its long-term future, particularly from mining and from lighting and development. One way forward has been through collaborative efforts with the Chilean government at local, regional, and national levels: in 2013 the Chilean government issued updated regulations for outdoor lighting in the region, which are beginning to be implemented. Another is to strengthen ties with the Chilean community through educational programs and outreach activities, in an effort both to get the public excited about the science and the wonders of the Universe and also to sensitize them to the fragile condition of the dark skies and the threats those skies face. Similar programs and activities are also being undertaken by other international observatories in Chile, including the European Southern Observatory, Carnegie Observatory, and the Giant Magellan Telescope.

Recently the AURA-O site was recognized as the first international Dark Sky Sanctuary by the International Dark-Sky Association, in recognition of the importance of the dark skies in northern Chile and the efforts to protect them. These efforts of international recognition are being furthered by the Chilean government through its initiative to propose key astronomical sites in northern Chile as UNESCO World Heritage sites. Such initiatives and collaborations aim to ensure that the skies of northern Chile remain dark for decades, if not centuries, to come.

\section{Global efforts to protect observatories from light pollution and/or radio frequency interference}

Pedro Sanhueza et al. (Highlights of the new Emission Norm for the Regulation of Light Pollution in Northern Chile) described efforts to address shortcomings in the first lighting regulations passed in Chile in 1998 (DS 686/1998) regarding scattering and overillumination, and to deal with the new menace of LEDs.

A new version of the regulations, developed by OPCC in collaboration with the Chilean 
Ministry of Environment (MMA), was approved by Presidential decree (DS 043/2012) in May 2013. This new environmental standard includes the following main restrictions:

- A full-cut-off requirement for general lighting: a maximum of $0.49 \mathrm{~cd} / \mathrm{KLm}$ at 90 degrees (i.e., no light distribution above the horizontal).

- For sport and recreational activities, an allowed level of $10 \mathrm{~cd} /$ Klumen at 90 degrees, together with a visor to cut upper-hemisphere emissions.

- Spectral restrictions divided into three regulated regions of the visible spectrum (as compared to the total light emission between 380 and $780 \mathrm{~nm}$ ):

- not more than $15 \%$ of total light emission in the range 300 to $380 \mathrm{~nm}$;

- not more than $15 \%$ in the range 380 to $499 \mathrm{~nm}$; and

- not more than $50 \%$ in the range $781 \mathrm{~nm}$ to $1000 \mathrm{~nm}$.

- Overillumination restricted to not more than $20 \%$ over the Chilean standard (NSEG $9 \mathrm{n} 71$ ) for minimal levels in public lighting.

- Billboards with inner sources of illuminations (LED or large plasma screens) must emit no more than $50 \mathrm{~cd} / \mathrm{m}^{2}$ at night. No spectral restriction is applied.

- Externally illuminated billboards must have full cut-off and be installed horizontally.

This new lighting regulation has yet not come into force, owing to a delay in approving complementary technical protocols. Enforcement is also a critical issue, given that the institutional environmental framework in Chile is being modified.

For the future, the OPCC is working with both the Ministry of Public Works and also the Ministry of Housing, seeking to go beyond the new lighting regulation by applying a stronger approach in terms of spectral restriction, promoting the use of warm white LEDs with a CCT of $2700 \mathrm{~K}$ and, in the case of outdoor illumination near professional observatories, monochromatic amber LEDs.

Richard Wainscoat (Protection of Hawaii's observatories from light pollution and radio frequency interference) explained that in the United States (as opposed to Chile or the Canary Islands), lighting ordinances are typically at the county or (occasionally) state level, meaning that astronomers have to deal with all levels of government: for example, military installations do not have to follow lighting ordinances.

The dark night sky over Mauna Kea - the largest collection of optical and infrared telescopes in the world - has been well protected by a strong lighting ordinance issued by Hawaii county, and remains very dark (Figure 2). Full shielding is now required for all new lights; there are now strict limits on blue light; and filtered LEDs are typically required for applications where white light is not required. The National Park Service night sky team visited Mauna Kea in July 2011, and found it to have a darker night sky than any of the US National Parks that they had visited. Astronomers have been working with Hawaii county's consultant on a revision of the Ordinance with, for example, widespread limitations on blue light. Enforcement is a key issue, with present enforcement being very lax, and the new draft includes new penalties for violations.

Haleakala Observatory on Maui-home to the Pan-STARRS telescopes, the Faulkes Telescope North, solar telescopes, and military telescopes - is more threatened, because Maui has a weaker lighting ordinance, and it is a smaller island, meaning that people live and work closer to the telescopes. Haleakala is also closer to Honolulu, and the urban glow from Honolulu contributes to an artificially bright sky towards the northwest (Figure 3).

Oahu has no lighting ordinance. The county is planning to replace all streetlights with $4000 \mathrm{~K}$ LEDs, most but not all of which will be fully shielded, but the use of $4000 \mathrm{~K}$, rather a warmer colour, is troubling.

As regards radio frequency interference, on Mauna Kea fixed radio transmitters are banned, as is Wi-fi, and cellular phones must be turned off. On Haleakala powerful TV 

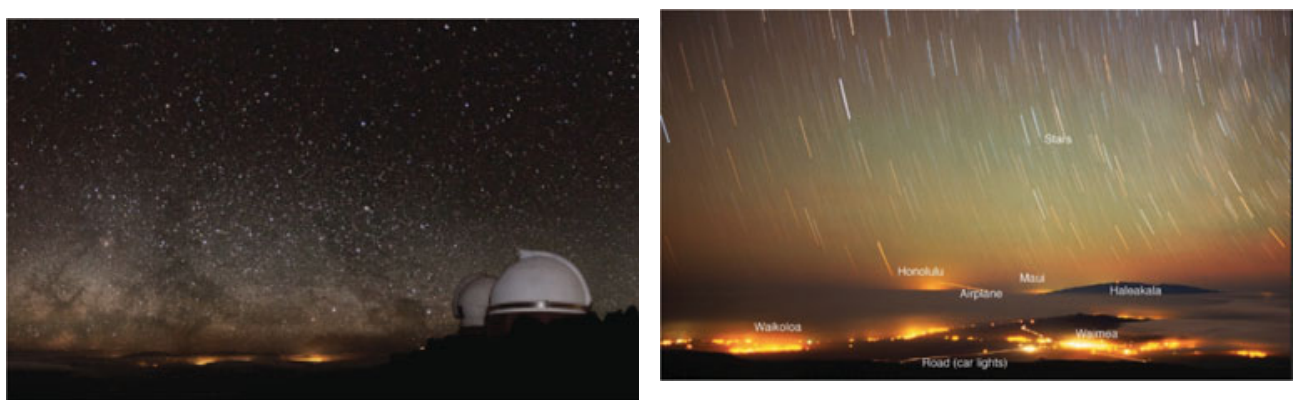

Figure 2: A view from Mauna Kea of the light from Waimea and Waikoloa (on the island of Hawaii)

Figure 3: A close up of light from Waimea and Waikoloa and Honolulu on Oahu, Haleakala and the island of Maui.

transmitters have been removed from the summit area. In both areas weather radar is sector blanked. On Haleakala there are still FAA radios and other government transmitters in the summit area, and there is little chance that these will be removed; however it is not believed that these interfere with Pan-STARRS.

Richard Green et al. (A Tale of Two Regions: Site Protection Experience and Updated Regulations in Arizona and the Canary Islands) compared experiences at two of the world's largest concentrations of telescopes containing some of the world's largest telescopes. Active site protection efforts are underway in both regions; the common challenge is to stay ahead of the LED revolution in outdoor lighting.

In Arizona, observatories and space science institutions are working together for site protection and economic leverage (Figure 4). Initial activities included drafting a model outdoor lighting code and the creation of presentation materials for the public and policy makers. The current focus is upon working with localities on lighting code updates and enforcement issues. A successful statewide conference was held in 2014 to raise awareness among public officials about issues of light pollution for astronomy, safety, wildlife and public health (see www.keystone.org/darkskies). There has been progress in improving lighting codes in the City of Tucson, smaller communities in northern Arizona (e.g. Winslow) and Sierra Vista, but there State-level battle in 2012 over a legislative bill to extend lighted signs into previously protected areas: the outcome was that the Phoenix metropolitan area can now have bright, animated LED signs, but they are prohibited in much of the rest of the State. Other major challenges include the fact that the city of Phoenix has invited proposals for 90,000 replacement street lighting fixtures at a $\mathrm{CCT}$ of $4000 \mathrm{~K}$, having rejected a request for the use of $3000 \mathrm{~K}$ (note the parallel to Honolulu). Another is that, as a result of current copper prices, the mining industry is seeking significant expansion. One contested development is at the foot of Mt. Hopkins (FL Whipple Obs. including MMTO) which would become the model lighting plant for outdoor mining operations, in violation of lighting protection zone limits; there will be a jurisdictional dispute.

In the Canary Islands, the 1988 "Sky Law" (31/1988) regulates light pollution, electromagnetic pollution, aircraft flight protected airspace, and air pollution. For the Oficina Técnica para la Protección de la Calidad del Cielo (OTPC), light pollution is the most time-consuming aspect: for example, the Canarian observatories are surrounded by National Parks and natural protected areas, so there are no air pollution problems. There is a range of legal lighting requirements, including no upward flux, switch-off or reduction 


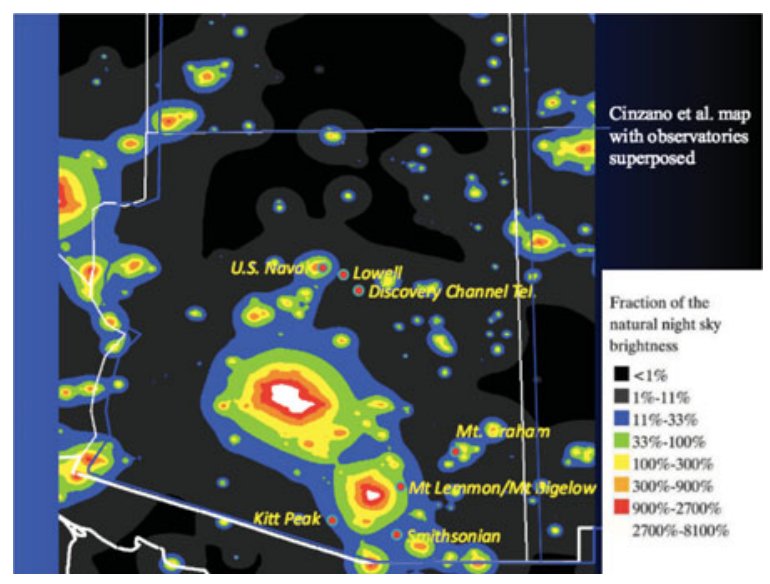

Figure 4: A map of Arizona from Cinzano et al.'s "World Atlas of the Artificial Night Sky Brightness" with observatory locations overlaid.

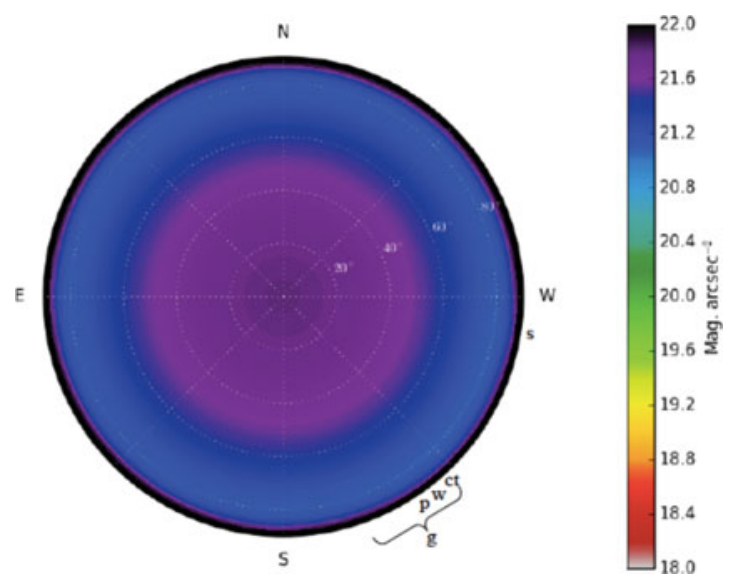

Figure 5: An all-sky image of night sky brightness in mag/sq arcsec. The "s", "ct", "w" and "p" denote the cities of Sutherland (14km from SAAO), Cape Town $(360 \mathrm{~km})$, Worcester $(254 \mathrm{~km})$, Paarl $(304 \mathrm{~km})$, respectively.

at midnight, and (generally) no emissions under 550nm. However, there are more flexible criteria for small pedestrian areas in gardens or surrounding buildings, and there are no colour restrictions on sport, advertisement and ornamental lighting. In the special case of La Palma, there are additional restrictions. The OTPC gives free technical advice, issues mandatory technical reports, certifies light devices, acts on complaints, collaborates with public bodies and associations, and undertakes popular outreach.

Ramotholo Sefako (Protection of SAAO observing site against light and dust pollution) described some issues faced by the South African Astronomical Observatory (SAAO) observing station near Sutherland, one of the darkest sites in the world for optical and IR astronomy (Figure 5). It hosts and operates several facilities, including the Southern African Large Telescope (SALT) and a number of international robotic telescopes.

In South Africa, the Astronomy Geographic Advantage (AGA) Act of 2007 empowers the Department of Science and Technology (DST) to regulate activities that pose a threat 


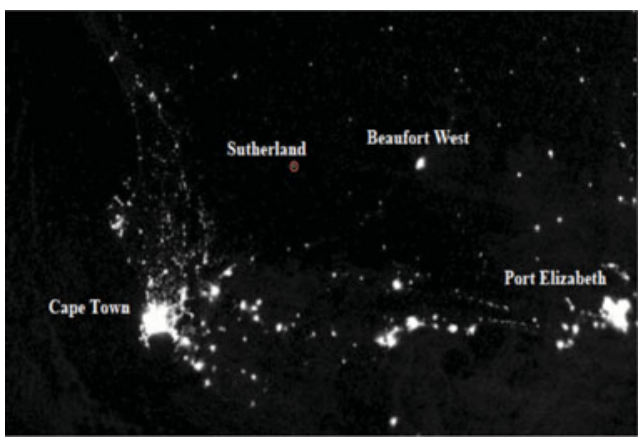

Figure 6: Nighttime image from orbit of Cape Town and cities near Sutherland. Cape Town is $360 \mathrm{~km}$ from Sutherland.

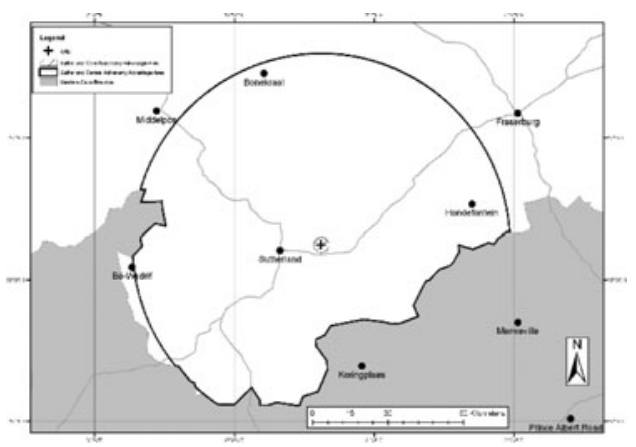

Figure 7: The Sutherland Central Astronomy Advantage Area (about 60 $\mathrm{km}$ radius). The Northern Cape and Western Cape provinces are shown in white and grey, respectively.

to optical and/or radio astronomy in areas declared Astronomy Advantage Areas (Karoo and Sutherland) (Figure 7). Such activities include mining or prospecting, outdoor lighting, power generation and "harmful industrial activities".

There are three tiers of protected areas:

- Core area - the physical area of the observatory or instrument;

- Central area - an area around the Core where the Minister can prohibit certain activities or categories of activity;

- Coordinated area - where the Minister sets standards with which activities must comply.

Challenges include planned developments around Sutherland, namely oil and gas exploration and exploitation (including fracking), mining, and wind farms. In particular, several wind farms are planned around Sutherland, within the central AAA; for example, a planned farm 20-25 km from the observatory proposes 50 wind turbines, lit, almost all of which will be visible from SALT/ Sutherland observatory. Astronomers continue to engage with different energy developers and local communities around the Sutherland Central AAA to find ways of protecting the observatory while at the same time permitting renewable energy developments.

Voluntary compliance continues to be the main strategy for protection of the observatory against light and dust pollution. This means promoting understanding and education: building good relationships with the public and the local community is an essential component of protecting South Africa's astronomical sites.

\section{A practical, heritage-based approach to the recognition of Dark Sky places as possible World Heritage sites}

Combining the perspectives of an astronomer and a heritage professional, Rémi Cabanac and Michel Cotte (Toward a Serial International Approach of the High Mountain Observatories, within important Dark Sky Value) considered how Outstanding Universal Value (OUV) might best be demonstrated in the case of a potential serial nomination of high-mountain observatories as "Windows to the Universe" 
that have maintained very important dark sky properties upon which their important astronomical functions depend.

The quality of the dark sky at a given place, and policies for its conservation, are very important, but insufficient in themselves to justify inscription on the World Heritage List. They must be related to important cultural or/and natural values, in other words significant cultural heritage features relating to astronomy and science and/or other exceptional natural attributes. The Dark Sky place must also demonstrate integrity/authenticity to an appropriate degree for today's tangible heritage of astronomy and have made a very significant contribution to international history of science and astronomy as an intangible attribute of the place. The last point must be demonstrated by a serious comparative analysis with similar places in the world and in the region. In the case of a serial nomination, each individual site must contribute significantly to the OUV of the global series.

The Pic du Midi Observatory in the French Pyrenees is likely to be an important component of such a nomination. It represents the early origin (at the end of the 19th century) of mountain scientific stations and observatories in Europe, with a long, continuous and important astronomical and scientific history lasting until the present with active programs of celestial and atmospheric observation.

\section{Further information}

Versions of the reports by M. Smith et al., C. Smith et al., and Sefako may be found in the FM2 pages in this volume. PDF versions of all the presentations in this session are available both on the FM2 pages on the UNESCO-IAU Portal to the Heritage of Astronomy (www.astronomical heritage.net/index.php/community/news-events/focusmeeting-at-iau-general-assembly) and on the FM21 pages on the NOAO website, wWW . noao.edu/education/IAUGA2015FM21.

\section{Reference}

Ruggles, C. \& Cotte, M. (eds.) 2015, Heritage sites of Astronomy and Archaeoastronomy in the Context of the UNESCO World Heritage Convention: Volume II (Bognor Regis: Ocarina Books), in press 